\title{
BRCA1 expression and improved survival in ovarian cancer patients treated with intraperitoneal cisplatin and paclitaxel: a Gynecologic Oncology Group Study
}

J L Lesnock ${ }^{1}$, K M Darcy ${ }^{2,8}$, C Tiann ${ }^{2,9}$, J A DeLoia ${ }^{3}$, M M Thrall ${ }^{4}$, C Zahn ${ }^{5}$, D K Armstrong ${ }^{6}$, M J Birrer $^{7}$ and T C Krivak ${ }^{*}, 1$

${ }^{1}$ Magee-Women's Hospital of UPMC, Division of Gynecologic Oncology, Pittsburgh PA 15213, USA; ${ }^{2}$ Gynecologic Oncology Group Statistical \& Data Center, Roswell Park Cancer Institute, Buffalo NY 14263, USA; ${ }^{3}$ Georgetown University Medical Center, Washington, DC 20057, USA; ${ }^{4}$ University of Washington, Seattle WA 98195-6460, USA; ${ }^{5}$ Uniformed Services University of the Health Sciences, Bethesda, MD 20814-4799, USA; ${ }^{6}$ Kimmel Cancer Center, Johns Hopkins School of Medicine, Baltimore MD 21231-1000, USA and ${ }^{7}$ Massachusetts General Hospital, Boston MA 02114, USA

Background: Breast cancer 1, early onset (BRCA1) is a tumour-suppressor gene associated with familial epithelial ovarian cancer (EOC). Reduced BRCA1 expression is associated with enhanced sensitivity to platinum-based chemotherapy. We sought to examine the prognostic relevance of BRCA1 expression in EOC patients treated with intraperitoneal platinum/taxane.

Methods: The GOG-172 was a phase III, multi-institutional randomised trial of intravenous paclitaxel and cisplatin (IV therapy) vs intravenous paclitaxel, intraperitoneal cisplatin plus paclitaxel (IP therapy) in patients with optimally resected stage III EOC. The BRCA1 expression was assessed with immunohistochemistry $(\mathrm{IHC})$ staining blinded to clinical outcome in archival tumour specimens. Slides with $\leqslant 10 \%$ staining were defined as aberrant and $>10 \%$ as normal. Correlations between BRCA1 expression and progression-free survival (PFS) and overall survival (OS) were analysed using Kaplan-Meier method and Cox regression analysis.

Results: Of the 393 patients, 189 tumours had aberrant expression, and 204 had normal BRCA1 expression. There was an interaction between BRCA1 expression and route of administration on OS $(P=0.014)$ but not PFS $(P=0.054)$. In tumours with normal BRCA1 expression, the median OS was 58 months for IP group vs 50 months for IV group $(P=0.818)$. In tumours with aberrant $B R C A 1$ expression, the median OS was 84 vs 47 months in the IP vs IV group, respectively $(P=0.0002)$. Aberrant BRCA1 expression was an independent prognostic factor for better survival in women randomised to IP therapy (hazard ratio $(H R)=0.67,95 \%$ confidence interval $(C I)=0.47-0.97, P=0.032$ ). Similar survival was observed in the IV and IP patients with normal BRCA1 expression. Multivariate but not univariate modelling demonstrated that IV patients with aberrant vs normal BRCA1 expression had worse survival.

Conclusion: Decreased BRCA1 expression is associated with a 36-month survival improvement in patients with EOC treated with IP chemotherapy. Although these results merit validation in future studies, the results suggest that decreased BRCA1 expression predicts for improved response to cisplatin-based IP chemotherapy with cisplatin and paclitaxel.

*Correspondence: Dr TC Krivak; E-mail: Tkrivak@mail.magee.edu

${ }^{8}$ Current address: Gynecologic Cancer Center of Excellence and the Women's Health Integrated Research Center at Inova Health System, Annandale, VA 22003, USA

${ }^{9}$ Current address: Precision Therapeutics Inc., Pittsburgh, PA 15213, USA

Received 24 October 2012; revised 26 January 2013; accepted 27 January 2013; published online 5 March 2013

(c) 2013 Cancer Research UK. All rights reserved 0007-0920/13 
Epithelial ovarian cancer (EOC) is estimated to affect 22200 women in the United States and lead to $\sim 15550$ deaths annually (Siegel et al, 2012). Women with EOC typically present with advanced disease and require aggressive cytoreductive surgery and chemotherapy to achieve remission and possible cure. Despite the fact that most patients will respond to initial therapy, the majority of patients will have recurrence. Ultimately, most of these patients with recurrent EOC die from the development of chemotherapyresistant disease (Cannistra, 2004; Lage and Denkert, 2007).

Breast cancer 1, early onset (BRCA1) is a tumour-suppressor gene located on chromosome $17 \mathrm{q} 21$ that has multiple roles in cellular functions. Germline mutations significantly increase a woman's risk of developing breast and/or EOC (Miki et al, 1994; Antonio et al, 2003). In sporadic EOC, BRCA1 dysfunction is frequently observed (Tutt and Ashworth, 2002). The BRCA1 protein combines with numerous proteins to regulate transcription, chromatin remodelling, ubiquitination and repair of doublestrand DNA breaks induced by DNA crosslinking agents, radiation or other exposures and events via homologous recombination (Tutt and Ashworth, 2002; Venkitaraman 2002; Yoshida and Miki, 2004; Weberpals et al, 2009). Given the various ways that BRCA1 protein expression can be altered in cancers, there is accumulating interest in the contribution of BRCA1 dysfunction on the pathogenesis of EOC and sensitivity to chemotherapy (Cass et al, 2003; Majdak et al, 2005; Wilcox et al, 2005; Chetrit et al, 2008). There are data to suggest that patients with reduced BRCA1 expression may have improved survival after platinum-based (or DNA damage-based) chemotherapy, yet reduced response to taxane-based (antimicrotubule) therapy (Foulkes, 2006).

The standard of care for patients with advanced EOC is treatment with a combination of platinum and taxane. Although a series of randomised phase III trials have demonstrated a dramatic survival advantage associated with IP (intravenous paclitaxel and cisplatin) vs IV (combination of intravenous paclitaxel and intraperitoneal cisplatin and paclitaxel) platinum and taxane chemotherapy, the community has not embraced IP therapy, in part because of toxicities and other concerns (Albert et al, 1996; Markman et al, 2001; Armstrong et al, 2006). The Gynecologic Oncology Group (GOG) has conducted another randomised phase III trial (GOG-252) to compare different platinum and taxanebased IP vs IV regimens in advanced-stage EOC and PPC. Currently, there is no test that can select which patients are likely to benefit from more aggressive and toxic IP therapy. Cisplatin induces intra- and interstrand crosslinks and will activate apoptosis if the damaged DNA is not adequately repaired. The IP administration of cisplatin produces a 12-fold greater drug exposure in the peritoneal cavity (Howel et al, 1982). We speculate that patients with aberrant tumour expression of BRCA1 expression will have a diminished ability to repair double-stranded DNA breaks and will therefore be more sensitive to IP therapy and will have better survival when compared with women with normal BRCA1 tumour expression.

We therefore undertook the following study to determine if BRCA1 pathway dysfunction, whether via germline mutations, somatic mutations or other mechanisms that result in decreased protein expression, helps define a group of patients who may respond to IP platinum-based chemotherapy. To this end, the concept of a common expression pattern that correlates with any BRCA dysfunction, ' $B R C A$ ness', was developed to define the role of BRCA function and response to cytotoxic chemotherapy (Turner et al, 2004; Tan et al, 2008). Konstaninopoulos et al (2010) reported improved responsiveness to platinum in a cohort of sporadic EOC patients with a BRCAness profile defined by gene expression analysis. BRCA1 expression can be assessed using immunohistochemistry (IHC), and studies have shown that absent/low BRCA1 protein expression can predict for an improved response to chemotherapy (Thrall et al, 2006; Swisher et al, 2008;
Carser et al, 2011). The primary objective of this study was to determine if the benefits of IP therapy, measured by progressionfree survival (PFS) and overall survival (OS), varied by the level of BRCA1 protein expressed in archival primary tumours from patients who participated in the randomised IP $v s$ IV phase III trial GOG-172 (Armstrong et al, 2006).

\section{MATERIALS AND METHODS}

Patient selection. Patients from GOG-172 protocol with archival tumour were eligible. The GOG-172 was a phase III, multiinstitutional, randomised trial of IV group $v s$ IP group for patients with a diagnosis of incident EOC or PPC (Armstrong et al, 2006). Inclusion criteria were patients with stage IIIC EOC or PPC with optimal, $<1 \mathrm{~cm}$ residual disease and one of the following histologic cell types: serous adenocarcinoma, endometrioid adenocarcinoma, mucinous adenocarcinoma, undifferentiated carcinoma, clear cell adenocarcinoma, mixed epithelial carcinoma, transitional cell, malignant Brenner's Tumour or adenocarcinoma not otherwise specified. The institutional review board at participating institutions approved the use of archival tissues as did the review board at Magee-Womens Hospital of the UPMC. All patients provided written informed consent consistent with all federal, state and local regulations before receiving treatment on GOG-172.

IHC for BRCA1 expression and scoring. Sections (5 $\mu \mathrm{m}$ thick) of primary tumour on glass slides were sent from each institution to the GOG Tissue Bank. The IHC assays for BRCA1 expression used the MS110 clone monoclonal antibody Ab-1 (Oncotech Inc., Tustin, CA, USA) that reacts with the N-terminal portion of the BRCA1 protein. This antibody has been utilised and validated by several authors (Thrall et al, 2006; Swisher et al, 2008; Mangia et al 2009). The MCF-7 cells were used as positive controls, whereas nonimmune serum was used as a negative control.

Percentage of staining was determined by two independent readers (JLL and TCK) and reviewed by a gynaecologic pathologist (CZ), all of whom were blinded to the identity of the samples and clinical outcome. The tumour specimens were scored in five separate locations throughout the slide, and multiple characteristics of staining were noted, including intensity of staining, location of staining (nuclear and/or cytoplasmic) and staining percentage.. The ascribed score was based on the number of cells with nuclear staining, specifically. Slides were scored as 0 if there was no staining, 1 if there was scattered staining $(<10 \%), 2$ if $10-50 \%$ of cells stained, 3 if $50-90 \%$ of cells stained and 4 if nearly all (>90\%) the cells were stained. Tumours were categorised as having aberrant BRCA1 expression for very low to no staining $(<10 \%$ staining; 0 or 1 score) and normal BRCA1 expression for $>10 \%$ BRCA1 staining (2-4 score). This scoring algorithm was developed and validated by Thrall et al (2006) and others (Swisher et al, 2008; Carser et al, 2011).

Statistical analysis. Progression-free survival was calculated as time of randomisation to either disease recurrence or death from any cause before recurrence, and OS was calculated from randomisation to death from any cause. The PFS and OS were estimated using the Kaplan-Meier method. The difference in survival function between patients with normal and aberrant BRCA1 expression was assessed by the log-rank test. Cox regression modelling was used to assess the association of BRCA1 expression and outcome using an unadjusted model and after adjusting for age, residual disease (microscopic vs gross), histological subtype and type of treatment (IP $v s$ IV). The hazard ratio (HR) was estimated for disease progression or death by BRCA1 expression. The interaction between BRCA1 expression and treatment was tested by extending the Cox model and adding an interaction term. The $\chi^{2}$ test (for categorical variables) or 
Wilcoxon rank-sum test (for continuous variables) was performed to evaluate the association of BRCA1 expression and patient characteristics. All reported $P$-values are two sided with $P<0.05$ considered statistically significant.

\section{RESULTS}

Of the 415 eligible patients enroled in GOG-172, archival formalinfixed and paraffin-embedded primary tumours were available from 393 patients, which represented $95 \%$ of all clinical trial participants. At the time of the current analysis, median follow-up for those who were still alive was 86 months. There were 282 patients who experienced disease progression and 255 who died. The lockout date for this study was similar to that used for the GOG-172 phase III trial (Armstrong et al, 2006).

Of the 393 patients in this study, 189 (48\%) tumours had aberrant and 204 (52\%) had normal BRCA1 expression. Patient characteristics were similar between the groups (Table 1). When the treatment regimen was ignored, aberrant $v s$ normal BRCA1 expression was associated with similar OS (Figure 1A) and PFS (Figure 1B). However, statistical evidence of an interaction was observed between tumour expression of BRCA1 and route of administration for OS $(P=0.014)$, suggesting that treatment efficacy for IP $v s$ IV was modified by expression level of BRCA1. Figure 2 illustrates the extent to which the OS (Figure 2A) and PFS (Figure $2 \mathrm{~B}$ ) varied by tumour expression of BRCA1 and route of administration of therapy. The IP-treated patients with aberrant BRCA1 expression had the best OS (Figure 2A) and PFS (Figure 2B). Median OS was 58 months for IP patients with normal BRCA1 expression vs 50 months for IV patients with normal BRCA1 expression $(P=0.818)$. In contrast, median OS was 84 months in the IP patients with aberrant BRCA1 expression $v s$ 48 months in IV patients with aberrant BRCA1 expression $(P=0.0002)$. Median PFS for IP patients with aberrant BRCA1 expression was 35 months compared with $<20$ months for the three other subgroups. However, the interaction between level of BRCA expression (aberrant $v$ s normal) and PFS did not achieve statistical significance $(P=0.054)$.

The influence of the route of administration on the relationship between BRCA1 expression and patient outcome was also assessed using unadjusted and adjusted Cox regression survival analysis (Table 2). Similar survival was observed for IP $v s$ IV patients with normal BRCA1 expression (unadjusted $\mathrm{HR}=0.97,95 \%$ confidence interval $(\mathrm{CI})=0.69-1.37, P=0.862$; adjusted $\mathrm{HR}=0.98,95 \%$ $\mathrm{CI}=0.69-1.38, \quad P=0.895)$. Significantly better survival was observed in the subset of IP patients with aberrant BRCA1 expression (unadjusted $\mathrm{HR}=0.68,95 \% \mathrm{CI}=0.47-0.97, P=0.034$ ). Aberrant BRCA1 expression retained independent prognostic value for survival in the subset of patients randomised to IP therapy after adjusting for age, residual disease (microscopic vs gross) and histologic subtype with an adjusted $\mathrm{HR}=0.67$ (95\% CI $=0.47-0.97, P=0.0320$ ). Similar survival was observed for IV patients with aberrant $v s$ normal BRCA1 expression (unadjusted $\mathrm{HR}=1.30,95 \% \mathrm{CI}=0.93-1.81, P=-.121)$. Multivariate modelling adjusting for age, residual disease (microscopic $v s$ gross) and cell type demonstrated that IV patients with aberrant $v s$ normal BRCA1 expression exhibited an increased risk of death (adjusted $\mathrm{HR}=1.46,95 \% \mathrm{CI}=1.04-2.03, P=0.028$ ).

\section{DISCUSSION}

In our study, we evaluated BRCA1 protein expression via IHC in tumour specimens from patients enroled in GOG-172 with respect to PFS and OS. Utilising IHC expression for BRCA1 in this study,

\begin{tabular}{|c|c|c|c|}
\hline Characteristic & $\begin{array}{c}\text { Aberrant } \\
(<10 \%) \text { BRCA1 } \\
\text { expression } \\
(n=189)\end{array}$ & $\begin{array}{c}\text { Normal } \\
(>10 \%) \text { BRCA1 } \\
\text { expression } \\
(n=204)\end{array}$ & $\boldsymbol{P}$-value \\
\hline \multicolumn{4}{|l|}{ Age (years) } \\
\hline Median (range) & $57.1(30.8-84.5)$ & $57.5(25.4-84.9)$ & 0.632 \\
\hline \multicolumn{4}{|l|}{ Race } \\
\hline $\begin{array}{l}\text { White } \\
\text { Black } \\
\text { Other }\end{array}$ & $\begin{array}{c}168(88.9) \\
6(3.2) \\
15(7.9)\end{array}$ & $\begin{aligned} 186 & (91.2) \\
5 & (2.4) \\
13 & (6.4)\end{aligned}$ & 0.749 \\
\hline \multicolumn{4}{|l|}{ GOG PS } \\
\hline $\begin{array}{l}0 \\
1 \\
2\end{array}$ & $\begin{array}{c}76(40.2) \\
102(54.0) \\
11(5.8)\end{array}$ & $\begin{array}{l}99(48.5) \\
94(46.1) \\
11(5.4)\end{array}$ & 0.249 \\
\hline \multicolumn{4}{|l|}{ Cell type } \\
\hline $\begin{array}{l}\text { Serous } \\
\text { Endometrioid } \\
\text { Clear cell } \\
\text { Mucinous } \\
\text { Others }\end{array}$ & $\begin{array}{c}151(79.9) \\
13(6.9) \\
10(5.3) \\
1(0.5) \\
14(7.4)\end{array}$ & $\begin{array}{c}164(80.4) \\
15(7.4) \\
8(3.9) \\
2(1.0) \\
15(7.4)\end{array}$ & 0.952 \\
\hline \multicolumn{4}{|l|}{ Tumour grade } \\
\hline $\begin{array}{l}1 \\
2 \\
3\end{array}$ & $\begin{array}{r}19(10.1) \\
70(37.0) \\
100(52.9)\end{array}$ & $\begin{array}{r}23(11.3) \\
78(38.2) \\
103(50.5)\end{array}$ & 0.867 \\
\hline \multicolumn{4}{|c|}{ Tumour residual } \\
\hline $\begin{array}{l}\text { Microscopic } \\
\text { Gross }\end{array}$ & $\begin{array}{r}75(39.7) \\
114(60.3)\end{array}$ & $\begin{array}{r}70(34.3) \\
134(65.7)\end{array}$ & 0.270 \\
\hline \multicolumn{4}{|l|}{ Treatment } \\
\hline $\begin{array}{l}\text { IV } \\
\text { IP }\end{array}$ & $\begin{array}{l}93(49.2) \\
96(50.8)\end{array}$ & $\begin{array}{r}107(52.5) \\
97(47.6)\end{array}$ & 0.520 \\
\hline
\end{tabular}

we have demonstrated that in this cohort of optimally resected patients with stage III EOC or PPC, aberrant BRCA 1 expression showed an improved PFS and OS when compared with patients whose tumours had normal BRCA 1 expression when stratified by treatment type. In patients whose tumours have aberrant BRCA1 expression, a clinically significant benefit in PFS and OS, 14 and 36 months respectively, was seen when those patients were treated with IP chemotherapy compared with patients whose tumours had normal expression of BRCA1 (Figure 2A and B). In patients whose tumours had normal expression of BRCA1, no significant improvement in PFS or OS with IV therapy was seen (Figure $2 \mathrm{~A}$ and $\mathrm{B}$ ).

In genomic stability, BRCA1 has an important role. Altered BRCA1 expression and/or dysfunction may occur as a result of germline mutations, somatic mutations or epigenetic modifications. Our analysis showed a high percentage of patients with aberrant BRCA1 expression in their tumours (52\%). This rate is similar to the rate of BRCA1 expression (46\%) seen in the analysis by Thrall et al (2006) and that reported by Carser et al (2011) (41\%) (Swisher et al, 2008). Low levels of BRCA1 (aberrant) expression have been reported to vary between 34 and $90 \%$ in the literature (Russell et al, 2000; Thrall et al, 2006 Swisher et al, 2009). This amount of variation may be the result of differences in the 

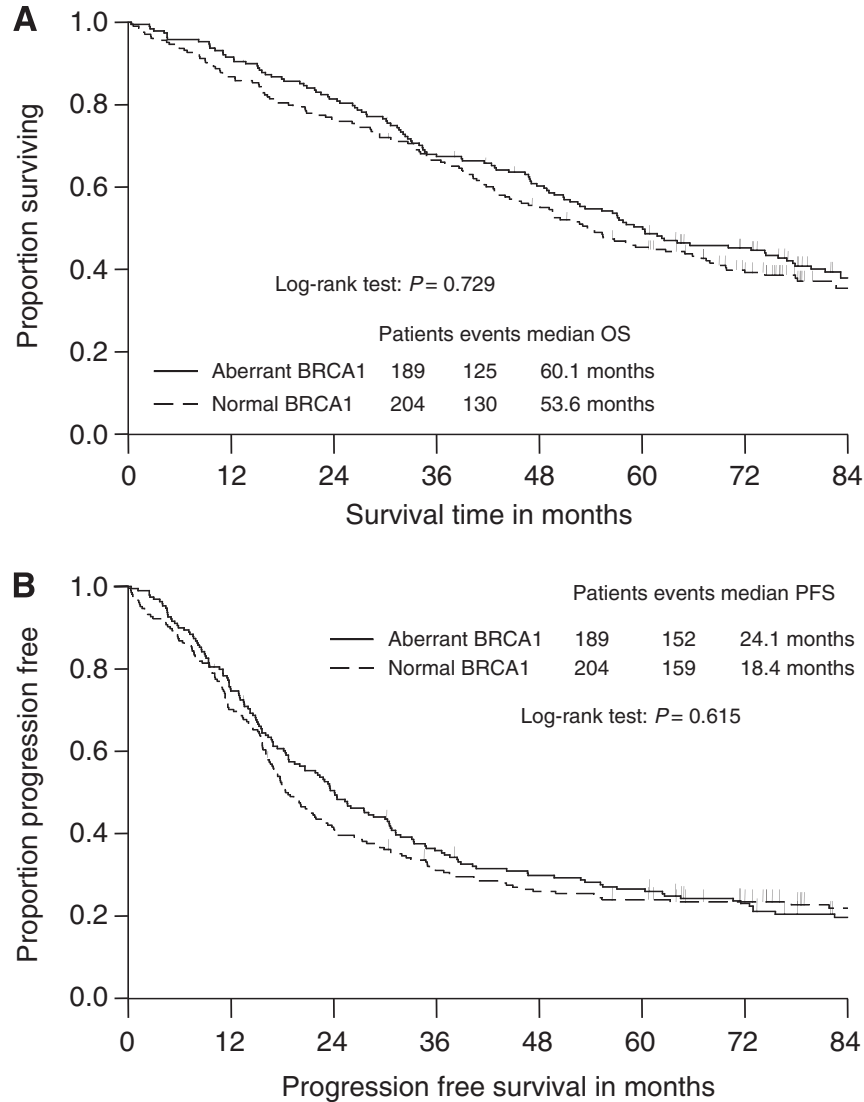

Figure 1. Kaplan-Meier estimate of (A) overall survival (OS) and (B) progression-free survival (PFS) for the entire cohort comparing aberrant with normal BRCA1 expression. Log-rank test was used to compare survival distributions across subgroups.
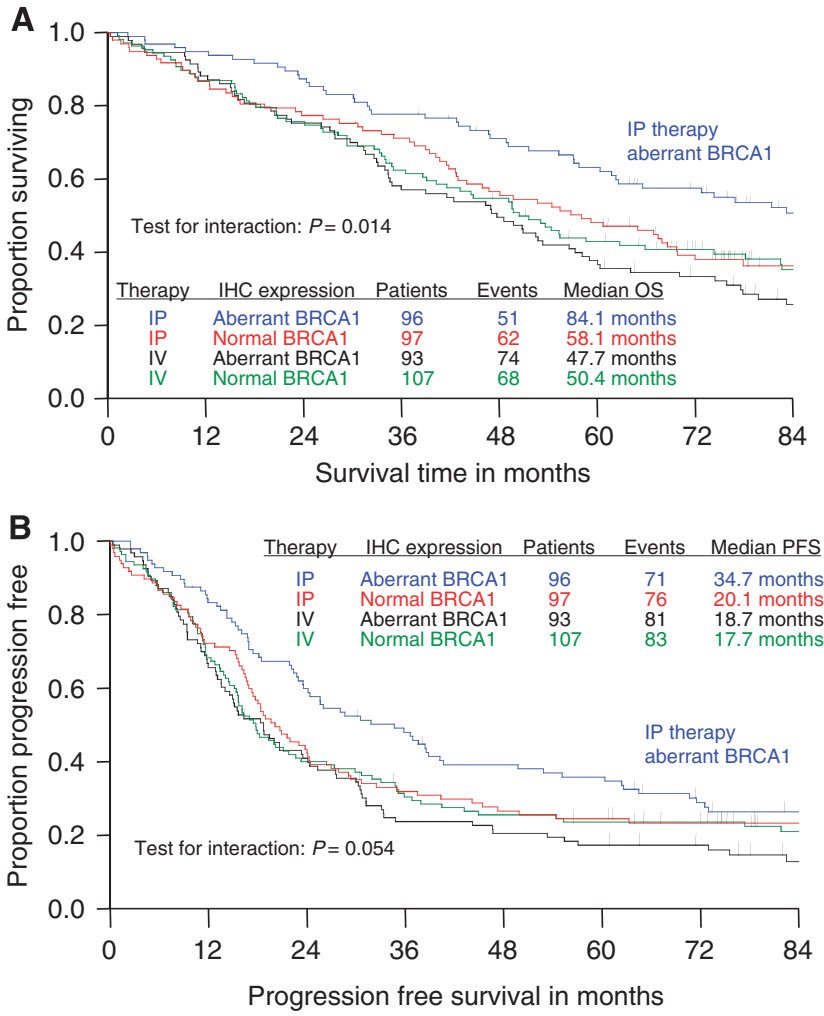

Figure 2. Kaplan-Meier estimate of (A) overall survival (OS) and (B) progression-free survival (PFS) for aberrant vs normal BRCA1 expression and by intraperitoneal (IP) vs intravenous (IV) therapy.

Table 2. Unadjusted and adjusted Cox regression modelling for PFS and OS for tumour expression of BRCA1 protein (normal vs aberrant) and type of therapy (IV vs IP)

\begin{tabular}{|c|c|c|c|c|}
\hline \multirow[b]{2}{*}{ Group } & \multicolumn{2}{|c|}{ Univariate analysis } & \multicolumn{2}{|c|}{ Multivariate analysis $^{a}$} \\
\hline & $\mathrm{HR}(95 \% \mathrm{Cl})$ & $\boldsymbol{P}$-value & $\mathrm{HR}(95 \% \mathrm{Cl})$ & $\boldsymbol{P}$-value \\
\hline \multicolumn{5}{|l|}{ PFS } \\
\hline IV patients with normal BRCA1 & Referent & & Referent & \\
\hline IV patients with aberrant BRCA1 & $1.19(0.88-1.62)$ & 0.263 & $1.33(0.98-1.81)$ & 0.072 \\
\hline IP patients with normal BRCA1 & $0.97(0.71-1.32)$ & 0.824 & $0.95(0.69-1.30)$ & 0.745 \\
\hline IP patients with aberrant BRCA1 & $0.74(0.54-1.02)$ & 0.065 & $0.72(0.52-0.99)$ & 0.043 \\
\hline \multicolumn{5}{|l|}{ os } \\
\hline IV patients with normal BRCA1 & Referent & & Referent & \\
\hline IV patients with aberrant BRCA1 & $1.30(0.93-1.81)$ & 0.121 & $1.46(1.04-2.03)$ & 0.028 \\
\hline IP patients with normal BRCA1 & $0.97(0.69-1.37)$ & 0.862 & $0.98(0.69-1.38)$ & 0.895 \\
\hline IP patients with aberrant BRCA1 & $0.68(0.47-0.97)$ & 0.034 & $0.67(0.47-0.97)$ & 0.032 \\
\hline
\end{tabular}

stage of disease, residual disease status, sample size, IHC assay conditions, interpretation of expression and treatment. Jazzeri et al (2002) compared gene expression profiles in BRCA1/2-associated EOC with sporadic EOC and showed that sporadic tumours may have mutations that resemble the molecular pathways seen in germline mutations as well as dysfunction in the downstream effects of these genes. The cancer genome atlas project (TCGA) recently published the genomic analysis of serous epithelial ovarian cancer. In this study, BRCA $1 / 2$ dysfunction was defined as germline mutations, somatic mutations and methylation in BRCA1. The results showed that in $33 \%$ of patients with advanced-stage serous ovarian cancer, BRCA1/2 dysfunction 
occurs and may provide a target for therapeutic interventions. In fact, $\sim 50 \%$ of cases exhibited defects in homologous recombination (The Cancer Genome Research Network, 2011). These levels are consistent with the $52 \%$ of cases with aberrant BRCA1 expression. Further testing will be required to confirm that EOC/ PPC patients with BRCA1-dependent defects in homologous recombination exhibit enhanced responsiveness and outcomes following IP therapy with cisplatin and paclitaxel. Additional studies will be required to determine if patients with BRCA1independent defects in homologous recombination benefit from IP vs IV platinum-based therapy, and whether the benefits from cisplatin IP-based therapy in women with aberrant BRCA1 expression extends to IP carboplatin as well.

Studies have also shown that patients who have BRCA1 germline mutations and develop EOC may have improved survival when compared with patients with sporadic EOC (Pharaoh et al, 1999; Pal et al, 2007; Tan et al, 2008). In our current study we evaluated PFS and OS with respect to BRCA1 IHC expression and stratified by chemotherapy delivery method (IV vs IP). We have shown that patients whose tumours have aberrant BRCA1 expression and were treated with IP cisplatin have a 36-month improvement in OS. This finding in patients whose tumours had aberrant BRCA1 expression and were treated with IP chemotherapy is biologically plausible. In several DNA repair mechanisms including homologous recombination, chromatin remodelling, regulation of the cell cycle and transcription, BRCA1 is implicated (Venkitaraman 2002; Yoshida and Miki 2004; Weberpals et al, 2009). These cellular functions are critical factors in how cells respond to cytotoxic agents, and particularly platinum. There are data to suggest that loss of BRCA1 function confers enhanced chemosensitivity to platinum-based chemotherapy and increase in resistance to antimicrotubule agents (Quinn et al, 2007; Stordal and Davey, 2009). Stordal and Davey (2009) recently reviewed a wide range of cell models and showed that modifications in BRCA1 (both increases and decreases) had the highest rate of inverse cisplatin/paclitaxel resistance phenotype compared with other gene mutations. As such, the role of BRCA1 dysfunction as related to chemotherapy response and patient survival is an important component in treatment planning for patients with EOC (Quinn et al, 2009). The IP therapy delivers higher concentrations of cytotoxic chemotherapy (Dedrick et al, 1978; Howel et al, 1982). Howel et al (1982)) demonstrated 12-fold higher peritoneal concentrations of cisplatin relative to plasma levels with intraperitoneal administration, and aberrant BRCA1 expression implies impaired DNA repair mechanisms. The additive effect of these mechanisms, more intensive and prolonged exposure to platinum in the setting of defective double-strand break repair, may explain why tumour cells with low expression of BRCA1 will be more sensitive to IP chemotherapy. In the subgroup analysis of this cohort, aberrant BRCA1 expression confers a survival benefit for patients treated with IP therapy, with a median PFS and OS of 35 and 84 months, respectively, which extends even beyond the survival advantage seen with IP therapy reported by Armstrong et al (2006). This suggests that tumour BRCA1 expression may be an important biomarker in sporadic EOC in determining response to IP chemotherapy and may provide a way to select ideal candidates for IP therapy.

These data also showed similar survival in IV and IP patients whose tumour exhibited normal BRCA1 expression. Worse survival was observed for IV patients with aberrant BRCA1 expression using multivariate but not using unadjusted univariate modelling. The negative effect of aberrant BRCA1 expression on OS required adjustments for age, residual disease and cell type. It should be noted that the proportion of cases with BRCA1 aberrant did not vary significantly by age, residual disease status or cell type. The improved survival seen with normal $v s$ aberrant expression of BRCA1 may be related to the BRCA1 effect on response to taxanes (low levels are associated with reduced response to taxanes). Normal BRCA1 expression may imply robust DNA repair, and therefore neutralising the clinical benefit of high intratumoural drug dose levels of platinum following IP therapy. Yang et al (2011) reported on BRCA1/2 expression and patient outcomes from the publically available Cancer Genome Atlas Project. This analysis demonstrated that only patients with BRCA2 germline mutations had improved OS (Yang et al, 2011). The 3-month difference for OS, although statistically significant, is not likely clinically significant. As patients with normal BRCA1 exhibit similar outcome following IV or IP therapy, consideration might be given to directing these patients to IV therapy and thus avoiding the extra toxicities associated with IP therapy, as these patients are unlikely to have the same clinical benefit from IP chemotherapy as those whose tumours have aberrant levels of BRCA1.

There are a few studies assessing BRCA1 expression via IHC as a prognostic marker for survival in patients with advanced-stage EOC (Thrall et al, 2006; Sirisabya et al, 2007; Swisher et al, 2008; Carser et al, 2011). Sirisabya et al (2007) reported on 99 patients with EOC, stages I-IV, and showed a positive BRCA1 IHC expression rate of $12 \%$ (defined as staining of $>10 \%$ ). Their results did not demonstrate any difference in PFS or OS; however, the authors did not stratify groups with respect to stage or residual disease. Swisher et al (2008) evaluated 115 sporadic EOC and reported a survival benefit in patients whose tumours had low BRCA1 expression. Thrall et al (2006) evaluated BRCA1 IHC expression relative to stage and histology in a subset of patients enroled in GOG-172. Of the 152 patients with outcome data, patients with low BRCA1 expression had an improved PFS and OS with a follow-up of 60 months. However, because of the limited sample size and follow-up, it was not designed to evaluate the impact of BRCA1 expression on PFS and OS. Finally, (Carser et al (2011) performed BRCA IHC on 292 ovarian tumours and showed an improved response to single-agent platinum with absent/low $(<10 \%)$ BRCA1 expression. Our report includes the largest series of patients enroled in a randomised trial $(n=393)$ with mature outcome data (median follow-up time of 86 months) using BRCA IHC. Additionally, our results may be slightly different because of the fact that all of the patients in our study had optimally resected stage III EOC or PPC and had received combination therapy with cisplatin and paclitaxel.

There are limitations with our current study. Our data did not show an overall survival advantage in patients whose tumours exhibited aberrant BRCA1 expression, which is in contrast to some prior published results (Swisher et al, 2008; Carser et al, 2011). Although atypical, this finding cannot detract from the results of the subgroup analysis. We also recognise the limitations of the assay for protein expression, namely IHC, which is assessed in a semiquantitative manner by independent reviewers and utilises an antibody that recognises the $\mathrm{N}$-terminal region of BRCA1. This technique has been widely adopted for the assessment of protein expression in other proteins and tumours (oestrogen receptor, progesterone receptor and HER-2 in breast cancer, and MLH1 in colon cancer), and is run in several clinical, commercial and research laboratories. The clinical utility of BRCA1 expression assessment in patients with advanced-stage EOC warrants further validation. The results of this study provide provocation for its assessment in future trials in conjunction with other methods of evaluating BRCA1, including quantitative mass spectrometry (MS)-based platforms assessing protein mass, sequence and concentration as well as modifications, BRCA-1 binding proteins via immunoprecipitation followed by immunoblotting and MS and BRCA1 transcriptional regulation by chromatin immunoprecipitation followed by next-generation sequencing for an example. Also, EOC and PPC may be further studied to characterise the type of BRCA dysfunction including somatic vs germline mutations, 
methylation, nuclear localisation and export, ubiquitin-mediated degradation and chromatin remodelling.

In conclusion, our data demonstrate a significant 3-year improvement in OS for patients with advanced EOC whose tumours exhibit aberrant BRCA1 expression treated with IP therapy. These were patients enroled in a phase III trial, with a large sample size, and treatment in each protocol arm was uniform. The method of IHC used in this report is commercially available and easily reproducible. Our findings suggest that IHC assessment of BRCA1 expression should be prospectively studied as a guide for the decision to use IP chemotherapy, and has been incorporated in the recent randomised phase III trial of IP $v s$ IV in EOC and PPC conducted by the Gynecologic Oncology Group protocol, GOG 252. The significant increase in survival in patients with aberrant BRCA1 expression treated with IP chemotherapy suggests that we can make a substantial improvement in this specific group of patients. Additionally, patients whose tumours had normal BRCA1 expression did not show improved survival with IP chemotherapy. If these findings are confirmed, this group of patients could be spared the side effects of IP therapy. Although these results warrant validation, these data add to the growing literature supporting the prognostic importance of BRCA1 protein expression and its potential for clinical application.

\section{ACKNOWLEDGEMENTS}

This study was supported by National Cancer Institute grants to the Gynecologic Oncology Group (GOG) Administrative Office and the GOG Tissue Bank (CA 27469), the GOG Statistical and Data Center (CA 37517) as well as a grant from the Gynecologic Oncology Group/Ovarian Cancer Research Fund New Investigator Award (to TCK).

\section{REFERENCES}

Alberts DS, Lui PY, Hannigan EV, O’Toole R, Williams SD, Young JA, Franklin EW, Clarke-Pearson DL, Malviya VK, DuBeshter B (1996) Intraperitoneal cisplatin plus intravenous cyclophosphamide versus intravenous cisplatin plus intravenous cyclophosphamide for stage III ovarian cancer. $N$ Engl J Med 335: 1950-1955.

Antoniou A, Pharoah PD, Narod S, Risch HA, Eyfjord JE, Hopper JL, Loman N, Olsson H, Johannsson O, Borg A, Pasini B, Radice P, Manoukian S, Eccles DM, Tang N, Olah E, Anton-Culver H, Warner E, Lubinski J, Gronwald J, Gorski B, Tulinius H, Thorlacius S, Eerola H, Nevanlinna H, Syrjakoski K, Kallioniemi OP, Thompson D, Evans C, Peto J, Lalloo F, Evans DG, Easton DF (2003) Average risks of breast and ovarian cancer associated with BRCA1 and BRCA2 mutations detected in case series unselected for family history: a combined analysis of 22 studies. Am J Hum Genet 72: 1117-1130.

Armstrong DK, Bundy B, Wenzel L, Huang HQ, Baergen R, Lele S, Copeland LJ, Walker JL, Burger RA. Gynecologic Oncology Group (2006) Intraperitoneal cisplatin and paclitaxel in ovarian cancer. $N$ Engl J Med 354: 34-43.

Cannistra SA (2004) Cancer of the ovary. N Engl J Med 351: 2519-2529.

Carser JE, Quinn JE, Michie CO, O'Brien EJ, McCluggage WG, Maxwell P, Lamers E, Lioe TF, Williams AR, Kennedy RD, Gourley C, Harkin DP (2011) BRCA1 is both prognostic and predictive biomarker of response to chemotherapy in sporadic epithelial ovarian cancer. Gynecol Oncol 123: 492-498.

Cass I, Baldwin RL, Varkey T, Moslehi R, Narod SA, Karlan BY (2003) Improved survival in women with BRCA-associated ovarian carcinoma. Cancer 97: 2187-2195.

Chetrit A, Hirsch-Yechezkel G, Ben-David Y, Lubin F, Friedman E, Sadetzki S (2008) Effect of BRCA1/2 mutations on long term survival in patients with advanced ovarian cancer; the national Israeli study of ovarian cancer. J Clin Oncol 26: 20-25.
Dedrick RL, Myers CE, Bungay PM, DeVita VT (1978) Pharmacokinetic rationale for peritoneal drug administration in the treatment of ovarian cancer. Cancer Treat Rep 62: 1-11.

Foulkes WD (2006) BRCA1 and BRCA2: chemosensitivity, treatment outcomes and prognosis. Fam Cancer 5: 135-142.

Howell SB, Pfeifle CL, Wung WE, Olshen RA, Lucas WE, Yon JL, Green M (1982) Intraperitoneal cisplatin with systemic thiosulfate protection. Ann Intern Med 97: 845-851.

Jazaeri A, Yee C, Sotriou C, Brantley K, Boyd J, Liu E (2002) Gene expression profiles of BRCA1-linked, BRCA2-linked, and sporadic ovarian cancers J Natl Cancer Inst 94: 990-1000.

Konstantinopoulos P, Spentzos D, Karlan B, Taniguchi T, Fountzilas E, Francoer N, Levine DA, Cannistra SA (2010) Gene expression profile of BRCAness that correlates with responsiveness to chemotherapy and with outcome in patients with epithelial ovarian cancer. J Clin Oncol 28: 3555-3561.

Lage H, Denkert C (2007) Resistance to chemotherapy in ovarian carcinoma. Recent Results Cancer Res 176: 51-1760.

Majdak EJ, Debniak J, Milczek T, Cornelise CJ, Devilee P, Emerich J, Jassem J, De Bock GH (2005) Prognostic impact of BRCA1 pathogenic and BRCA1/ BRCA2 unclassified variant mutations in patients with ovarian carcinoma. Cancer 104: 1004-1012.

Mangia A, Chiriatti A, Tommasi S, Menolascina F, Petroni S, Zito FA, Simone G, Schittulli F, Paradiso A (2009) BRCA1 expression and molecular alterations in familial breast cancer. Histol Histopathol 24: 69-76.

Markman M, Bundy BN, Alberts DS, Fowler JM, Clark-Pearson DL, Carson LF, Wadler S, Sickel J (2001) Phase III trial of standard dose intravenous cisplatin plus paclitaxel versus moderately high-dose carboplatin followed by intravenous paclitaxel and intraperitoneal cisplatin in small volume stage III ovarian carcinoma: an intergroup study of the Gynecologic Oncology Group, Southwestern Oncology Group, and Eastern Cooperative Oncology Group. J Clin Oncol 19: 1001-1007.

Miki Y, Swensen J, Shattuck-Eidens D, Futreal PA, Harshman K, Tavtigian S, Liu Q, Cochran C, Bennett LM, Ding W (1994) A strong candidate for the breast and ovarian cancer susceptibility gene BRCA1. Science 226: 66-71.

Pal T, Permuth-Wey J, Kapoor R, Cantor A, Sutphen R (2007) Improved survival in BRCA2 carriers with ovarian cancer. Fam Cancer 6: 113-119.

Pharaoh PD, Easton DF, Stockton DL, Gayther S, Ponder BA (1999) Survival in familial BRCA1- associated, BRCA2- associated epithelial ovarian cancer. United Kingdom Coordinating Committee for Cancer Research (UKCCCR) Familial Ovarian Cancer Study Group. Cancer Res 59: 868-871.

Quinn JE, Carser JE, James CR, Kennedy RD, Harkin DP (2009) BRCA1 and implications for response to chemotherapy in ovarian cancer. Gynecol Oncol 113: 134-142.

Quinn JE, James CR, Stewart GE, Mulligan JM, White P, Chang GK, Mullan PB, Johnston PG, Wilson RH, Harkin DP (2007) BRCA1 mRNA expression levels predict for overall survival in ovarian cancer after chemotherapy. Clin Cancer Res 13: 7413-7420.

Russell PA, Pharoah PD, de Foy K, Ramus SJ, Symmonds I, Wilson A, Scott I, Ponder BA, Gayther SA (2000) Frequent loss of BRCA1mRNA and protein expression in sporadic ovarian cancers.. Int J Cancer 87: 317-321.

Siegel R, Naishadham D, Jemal A (2012) Cancer statistics, 2012. CA Cancer J Clin 62: 10-29.

Sirisabya N, Manchana T, Termrungreunglert W, Triratanachat S, Charuruks N, Tresukosol D (2007) Prevalence of BRCA1 expression in epithelial ovarian cancer: immunohistochemical study. J Med Assoc Thai 90: 9-14.

Stordal B, Davey R (2009) A systematic review of genes involved in the inverse resistance relationship between cisplatin and paclitaxel chemotherapy: role of BRCA1. Curr Cancer Drug Targets 9: 354-365.

Swisher EM, Gonzalez RM, Taniguchi T, Garcia RL, Walsh R, Goff BA, Welsch P (2009) Methylation and protein expression of DNA repair genes: association with chemotherapy exposure and survival in sporadic ovarian and peritoneal carcinomas. Mol Cancer 8: 48-54.

Swisher EM, Sakai W, Karlan BY, Wurz K, Urban N, Taniguchi T (2008) Secondary BRCA1 mutations in BRCA1-mutated ovarian carcinomas with platinum resistance. Cancer Res 68: 2581-2586.

Tan D, Rothermundt C, Thomas K, Bancroft E, Eeles R, Shanley S, ArdenJones A, Norman A, Kaye SB, Gore ME (2008) "BRCAness" syndrome in ovarian cancer: a case control study describing the clinical features and outcomes of patients with epithelial ovarian cancer associated with BRCal and BRCA2 mutations. J Clin Oncol 26: 5530-5536. 
The Cancer Genome Research Network (2011) Integrated genome analyses of ovarian carcinoma. Nature 474(7353): 609-615.

Thrall M, Gallion HH, Kryscio R, Kapali M, Armstrong DK, Deloia JA (2006) BRCA1 expression in a large series of sporadic ovarian carcinomas: a Gynecologic Oncology Group Study. Int J Gynecol Cancer 16: 166-171.

Turner N, Tutt A, Ashworth A (2004) Hallmarks of "BRCAness" in sporadic cancers. Nat Rev Cancer 4: 814-819.

Tutt A, Ashworth A (2002) The relationship between the roles of BRCA genes in DNA repair and cancer predisposition. Trends Mol Med 8: 571-576.

Venkitaraman AR (2002) Cancer susceptibility and the functions of BRCA1 and BRCA2. Cell 108: 171-182.

Weberpals J, Garbuio K, O'Brien A, Clark-Knowles K, Doucett S, Antoniouk O, Glenwood G, Dimitroulakos J (2009) The DNA repair proteins BRCA1 and ERCC1 as predictive markers in sporadic ovarian cancer. Int $J$ Cancer 24: 806-815.
Wilcox CB, Basal BE, Gallion HH, Strange MA, Deloia JA (2005) Highresolution methylation analysis of the BRCA1 promotor in ovarian tumors. Cancer Genet Cytogenet 159: 114-122.

Yang D, Kahn S, Sun Y, Hess K, Shmulevich I, Sood AK, Zhang W (2011) Association of BRCA1 and BRCA2 mutations with survival, chemotherapy sensitivity, and gene mutator phenotype in patients with ovarian cancer. JAMA 306: 1557-1565.

Yoshida K, Miki Y (2004) Role of BRCA1 and BRCA2 as regulators of DNA repair, transcription, and cell cycle response to DNA damage. Cancer Sci 95: 866-871.

This work is published under the standard license to publish agreement. After 12 months the work will become freely available and the license terms will switch to a Creative Commons AttributionNonCommercial-Share Alike 3.0 Unported License. 